\title{
Detection Systems of Ultra-High-Resolution SEMs
}

\author{
Jaroslav Jiruše ${ }^{1}$, Miloslav Havelka ${ }^{1}$, Jan Polster $^{1}$, Petr Sytař ${ }^{1}$, Jan Páral ${ }^{1}$ and Jolana Kološová ${ }^{1}$ \\ 1. TESCAN Brno, Brno, Czech Republic.
}

For most current Scanning Electron Microscopes (SEM), the traditional Everhart-Thornley secondary electron (SE) detector and annular backscattered electron (BSE) detector are complemented with several detectors placed in the column. To choose the right system suitable for appropriate application is a challenging task. TESCAN introduced two ultra-high-resolution SEM columns [1], [2] in the last two years. Here, we present a comparison of both systems and introduce recent improvements. Both systems allow angular filtering of SE, energy-filtering of BSE and angular BSE selection.

The BrightBeam ${ }^{\mathrm{TM}}$ SEM column [1] combines a high-potential tube with a magnetic-electrostatic objective lens delivering ultra-high resolving power when the specimen is in the magnetic-field-free mode. Two specialized detectors in the SEM column together with in-chamber detectors enable the selection of multiple signals for angular SE and BSE (see Figure 1). The Multidetector acquires wide-angle SEs while the Axial detector collects the axial part of the SE signal responsible for enhanced surface sensitivity. Wide angle BSEs impact the BSE detector in the chamber and/or are converted to a SE3 signal with topography BSE information detected on the in-chamber E-T detector. The potential applied on the grid of the Multidetector allows for filtering out the low-energy signal electrons to collect narrow-angle BSEs with good Z-contrast or low-loss BSEs created in the top layer of the sample. The angular BSE filtration is completed with a dedicated imaging mode, where the Axial detector collects the portion of the BSE signal close to the optical axis, which is shown to yield increased surface sensitivity with minimized topography at higher beam energies.

The Triglav ${ }^{\mathrm{TM}}$ SEM column [2] is equipped with the TriLens ${ }^{\mathrm{TM}}$ triple objective lens system to enable ultra-high resolution imaging when the specimen is immersed in the magnetic field, analysis in field-free mode and multiple display modes combining all three lenses. The detection system includes a triplet of complementary BSE detectors (TriBE ${ }^{\mathrm{TM}}$ ) that provides angular selection of BSEs (see Figure 2). Inchamber BSE with enhanced sensitivity in the low-energy region (down to $200 \mathrm{eV}$ ) provides a strong signal as well as mixed material and topographic contrast due to wide-angle BSEs. The Mid-angle detector placed in the column utilizes the conversion effect and has excellent signal at low energies $<2 \mathrm{keV}$. The imaging capabilities of the system are significantly enriched by adding a filtering grid to the In-Beam BSE detector collecting axial electrons, enabling surface-sensitive BSE imaging in the normal mode, as well as in the beam deceleration mode.

Sample topography is mapped using TriSE ${ }^{\mathrm{TM}}$ - a triplet of secondary electron detectors. The in-chamber $\mathrm{SE}$ detector is dedicated to observation in the analytical mode. In the ultra-high-resolution mode, secondary electrons are effectively driven into the column where they are attracted to the In-Beam SE detector. The upper in-column detector, SE (BDM), collects SE in the beam deceleration mode.

Since both columns are capable of ultra-high resolution imaging, the detection system is the main factor determining the choice of a device best-suited for any intended application. We have observed excellent image contrast while imaging biological samples with the BrightBeam ${ }^{\mathrm{TM}}$ column and semiconductor chips with the Triglav $^{\mathrm{TM}}$ column. 
References:

[1] J Jiruse, M Havelka and J Polster, Microscopy and Microanalysis 22 (2016), p. 578.

[2] P Sytar, J Jiruse and A Zavodny, Microscopy and Microanalysis 23 (2017), p. 38.

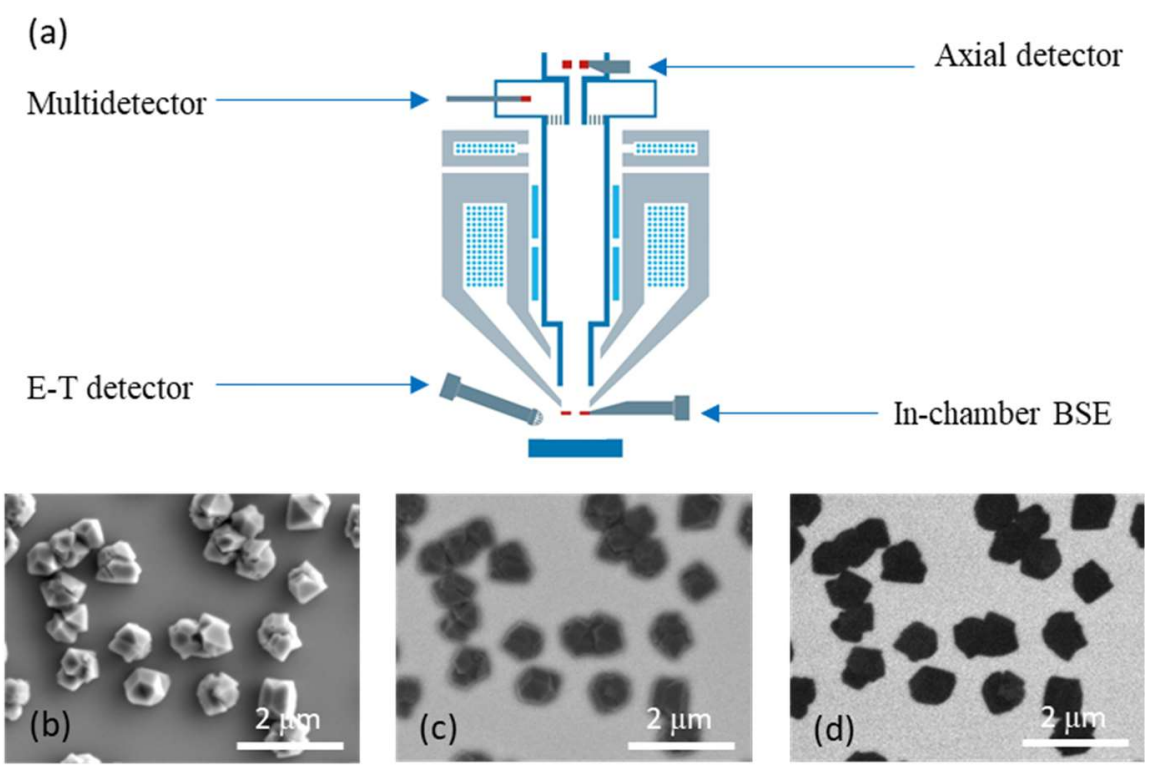

Figure 1. (a) Schematic of the detection system in the BrightBeam ${ }^{\mathrm{TM}}$ column: example of angular BSE selection (sample - diamond on silicon); (b) strong topographic contrast in the image from the E-T detector, (c) material contrast from the Multidetector, (d) surface-sensitive material contrast from the Axial detector.

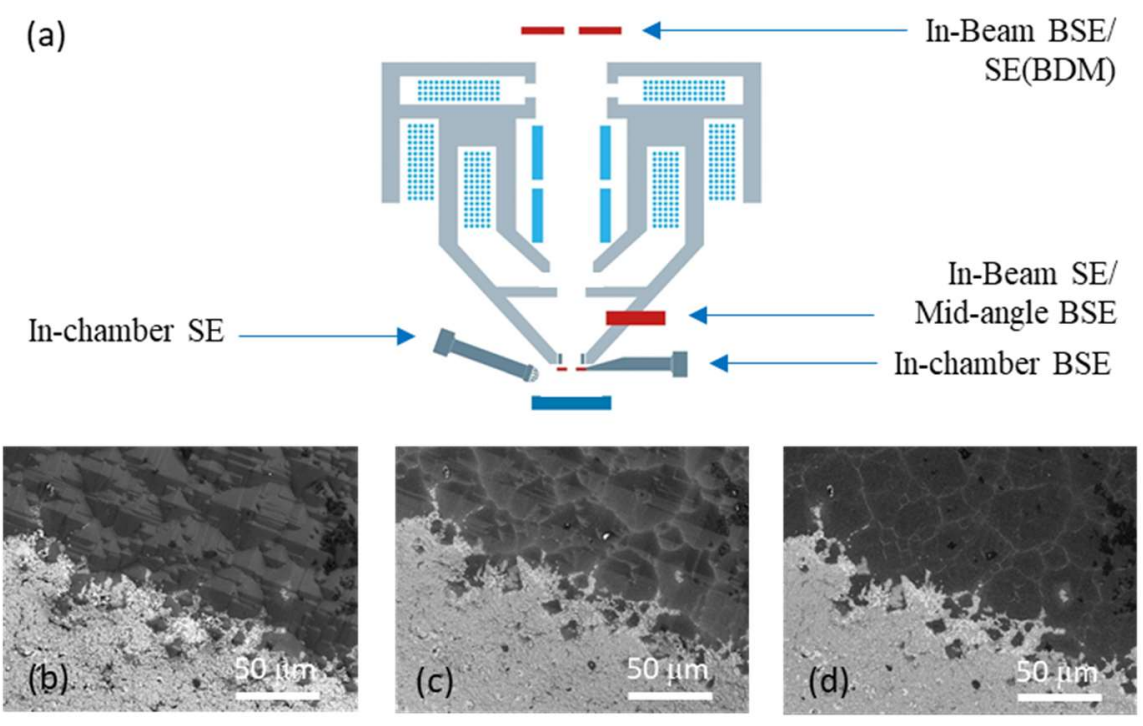

Figure 2. (a) Schematic of the detection system in the Triglav ${ }^{\mathrm{TM}}$ column: example of angular BSE selection (sample - SiN with Ag contacts); (b) topographic and Z-contrast in the image from in-chamber BSE detector, (c) Z-contrast from whole interaction volume from the Mid-Angle BSE detector, (d) Zcontrast from a thin surface layer from the In-Beam BSE detector. 